Research Article

\title{
Simulation Analysis of Cylindrical Shell Cavity Noise with Melamine Foam Lining
}

\author{
Menghui Qi $\left(\mathbb{D},{ }^{1}\right.$ Bin Li, ${ }^{2}$ Shilin Yan $\mathbb{D}^{1},{ }^{1}$ Qinghua Yan, ${ }^{2}$ and Qiyu $\mathrm{He}^{2}$ \\ ${ }^{1}$ Hubei Key Laboratory of Theory and Application of Advanced Materials Mechanics, Wuhan University of Technology, \\ Wuhan 430070, Hubei, China \\ ${ }^{2}$ School of Mechanical Engineering, Wuhan Polytechnic University, Wuhan 430023, Hubei, China \\ Correspondence should be addressed to Shilin Yan; yanshilin408@126.com
}

Received 26 October 2020; Revised 10 April 2021; Accepted 26 May 2021; Published 10 June 2021

Academic Editor: Jie Yang

Copyright (C) 2021 Menghui Qi et al. This is an open access article distributed under the Creative Commons Attribution License, which permits unrestricted use, distribution, and reproduction in any medium, provided the original work is properly cited.

To solve the problem of the harsh midlow frequency noise of rocket fairing, the cylindrical section of the protective precision instrument fairing is simplified as cylindrical shells, and different lining strategies of melamine foam (MF) are studied experimentally and numerically. Based on Virtual.Lab Acoustic software, a finite element model of the cylindrical cavity is established, and the correctness is verified by comparison with the measured data. On that basis, the influences of the lining position of different thickness MF on the noise reduction of cylindrical shells are investigated. It is shown that the thickness and location of the laying material have a significant effect on the noise reduction at the same specific gravity.

\section{Introduction}

In recent years, carrier rockets have been developing towards high thrust and high load ratio [1]. As a result, the noise environment in the cowling chamber of the rocket protecting the payload has become more severe, and the noise control in the midlow frequency in the cavity has become more urgent [2]. Because precision instruments and their payloads are usually placed in the fairing cylinder section, the internal sound field of the high-speed railway vehicle body, aircraft fuselage, and some heavy machinery can be converted into a similar noise reduction study in the fairing cylinder section cavity. Therefore, the acoustic vibration characteristics and the noise in the cylindrical shell cavity have attracted extensive attention of researchers [3-5].

Chonan and Koriyama [6] analyzed the sound insulation performance of finite length anisotropic cylindrical shell structure, and the results showed that the transmission loss in the cavity would be increased with decreasing radius and length of the cylinder structure. Ghinet et al. [7] calculated the model of sound transmission characteristics of interlayer infinitely long cylindrical shell in the reverberation field. Yao et al. [8] deduced the wave equation in uniform flow field based on the classical thin shell theory and analyzed the influence of incidence angle, damping thickness, and shell thickness on STL. Denli and Sun $[9,10]$ take the fiber lining direction as the design variable and increase the STL of a composite cylindrical shell as the optimization objective, which effectively improves the STL of the structure in the low-frequency region. Talebitooti et al. [11] performed an acoustic analysis of the four sides that simply supported doubly curved composite shell interlayered with porous materials. Valvano et al. [12] proposed a sound transmission analytical formulation considering fractional derivatives models for the viscoelastic damping for multilayered shells made of composite and viscoelastic layers.

The sound insulation performance of the cylindrical shell itself is very limited, so passive noise reduction is used to further enhance the noise reduction performance of the cylindrical shell. Valvano et al. [5] developed an accurate shell finite element model for the passive sound insulation analysis of composite laminated structures embedding viscoelastic layers. Engberg and Korde [13] took the cylinder section of a satellite fairing as the research object, established the cylindrical shell correlation model, and studied the influence law of acoustic overlay on the noise reduction effect. 
Chen et al. [14] studied the acoustic radiation characteristics of cylindrical shells lining viscoelastic materials and discussed the influence of the elastic modulus and loss factor of viscoelastic materials. Lane et al. [3] used experimental testing methods to study the effect of lining melamine foam on the inner wall on the acoustic response of the fairing cavity. Li et al. [15-17] studied the noise reduction of cylindrical cavity lining MF in midlow frequency. Yan et al. [18] studied the lining scheme of the acoustic cladding of cylindrical shells and the influence of lining thickness on noise response using the finite element simulation method. Gohari et al. [19] presented an analytical model to embed porous materials in a finite cylindrical shell to obtain the sound transmission loss coefficient.

In practical application, the quality of the lining materials in the cylindrical shell cavity is strictly limited. In other words, it is impossible to lay the noise-reducing materials all over the shell. Therefore, it is important to select the lining location with the best noise reduction effect when the lining rate keeps constant. This article selects a typical porous sound-absorbing material MF as the acoustic covering layer. Based on the experimental conditions, the influence of installation position and thickness of MF on the STL of the cylindrical shell in $100-400 \mathrm{~Hz}$ was studied. The results will provide a useful reference for further research on the applicable law and characteristics of acoustic overlay in passive noise control.

\section{Experimental Research}

2.1. Experimental Test. To obtain the sound pressure response values inside and outside of the cylindrical shell, a cylindrical shell noise experiment device was established. The cylindrical shell was placed in the center of the reverberation room, and the bottom is supported by four $50 \mathrm{~mm}$ shock absorbing rubber pads to mimic the ideal free state. Two loudspeakers were placed diagonally in the room to provide sound sources. The experimental sound source signal was a piece of white Gaussian noise, which was passed into the speaker through the power amplifier and finally released by the speaker after filtering by an equalizer. After the sound field was stabilized, the sound pressure values inside and outside the cylinder shell cavity were obtained.

The cylindrical shell was made of a $1 \mathrm{~mm}$ aluminum plate, with a height of $1125 \mathrm{~mm}$, an outer wall diameter of $1040 \mathrm{~mm}$, and a mass of $12.20 \mathrm{~kg}$. The thickness of internal reinforcing bars was $1.50 \mathrm{~mm}$. There were 6 circular stiffeners and 20 longitudinal stiffeners; the internal expansion diagram of the cylindrical shell cut along the longitudinal ribs is shown in Figure 1. The upper and lower end faces of the cylindrical shell were sealed with wood boards, with a diameter of $1180 \mathrm{~mm}$ and a thickness of $25 \mathrm{~mm}$. A columnar depression with a diameter of $1040 \mathrm{~mm}$ and a depth of $12.5 \mathrm{~mm}$ was set in the middle of the wood boards as shown in Figure 2.

There were 27 acoustic sensors in the experiment, of which three were located in the middle position outside the cylinder shell and one was placed every $120^{\circ}$ from the shell $10 \mathrm{~mm}$ to test the sound pressure level (SPL) of the external sound field. Two acoustic sensors were placed at $90^{\circ}$ intervals in the upper, middle, and lower three layers of the cylindrical shell cavity at radius $R$ and $0.5 R$, respectively, as shown in Figure 3. Eight sensors were placed in each layer to test the SPL in the cavity.

2.2. Experimental Test Results. The mean value of the data measured by the three acoustic sensors on the outer surface of the cylindrical shell was used as the external load, and the mean value of the data measured by the 24 acoustic sensors inside the cylindrical shell cavity was used as the internal response noise. The transmission loss can be calculated by the following formula:

$$
\mathrm{STL}=10 \log \left(\frac{W^{I}}{W^{T}}\right)=L_{W^{I}}-L_{W^{T}},
$$

where $W^{I}$ and $W^{T}$ are the incident and transmitted sound energy of the cylindrical shell; $L_{W^{I}}$ and $L_{W^{T}}$ are the incident and radiated sound power level, i.e., $L_{W^{I}}$ is the total SPL of load and $L_{W^{T}}$ is the total noise level in the shell cavity. Figure 4 illustrates that in the frequency range of $0-1000 \mathrm{~Hz}$, the total SPL of the load is $107.49 \mathrm{~dB}$, the total noise level in the shell cavity is $95.43 \mathrm{~dB}$, and the total STL of the cylindrical shell is $12.06 \mathrm{~dB}$. Therefore, the cylindrical shell has certain sound insulation and noise reduction effects in the frequency range of $0-1000 \mathrm{~Hz}$.

\section{Finite Element Simulation Analysis}

3.1. Establishment of the Simulation Model. Based on finite element software, a finite element model consistent with the cylindrical shell structure used in the experiment was established. The cylindrical shell and stiffeners were meshed by CQUAD tetrahedral element, the upper and lower cover plates were meshed by CHEXA tetrahedral element, and the stiffeners were connected with the rivets on the surface wall by RBE2 element mesh. As for the division of acoustic cavity mesh, CHEXA hexahedral element was used to simulate the acoustic cavity inside and outside the cylindrical shell. The entire simulation model contains 57897 structural elements and 162796 acoustic elements.

The model was imported into the acoustic finite element analysis module of LMS Virtual.Lab acoustic software, and corresponding grid properties and material properties were assigned to different elements, respectively. The material parameters used in the simulation are given in Table 1, and the MF acoustic parameters use the test results from Italian SCS Institute. MPC line welding constraints were adopted to connect the cylindrical shell with the upper and lower cover plates. The inner and outer sound cavities were set to couple with the shell and the inner surface of the upper and lower cover plate, and tolerance values of different coupling surfaces are set according to the model. The plane of the lower surface of the cylindrical shell was defined as the reflection surface, and the other outer surfaces of the finite element model are defined as the automatic matching layer.

To simulate the experimental reverberation sound field outside the shell, the reverberation source load (represented 


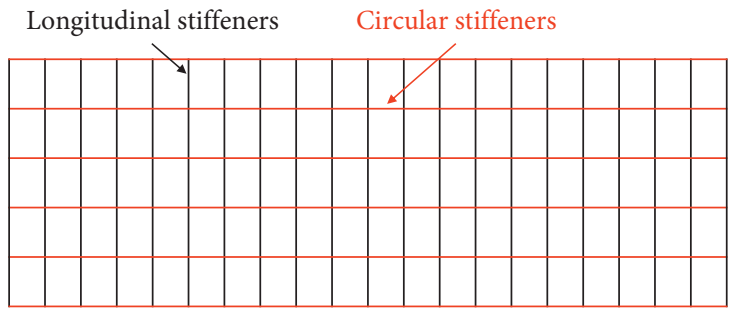

Figure 1: Schematic diagram of cylindrical shell wall expansion.

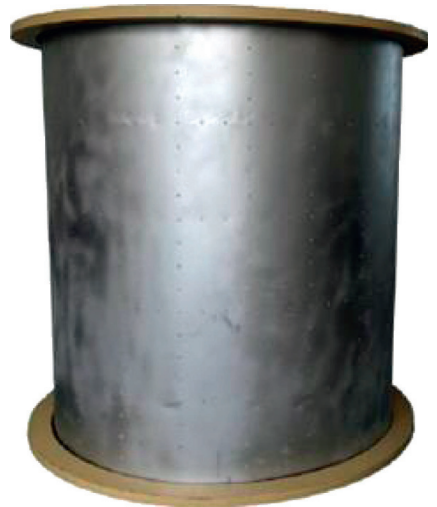

Figure 2: The cylindrical shell test model.

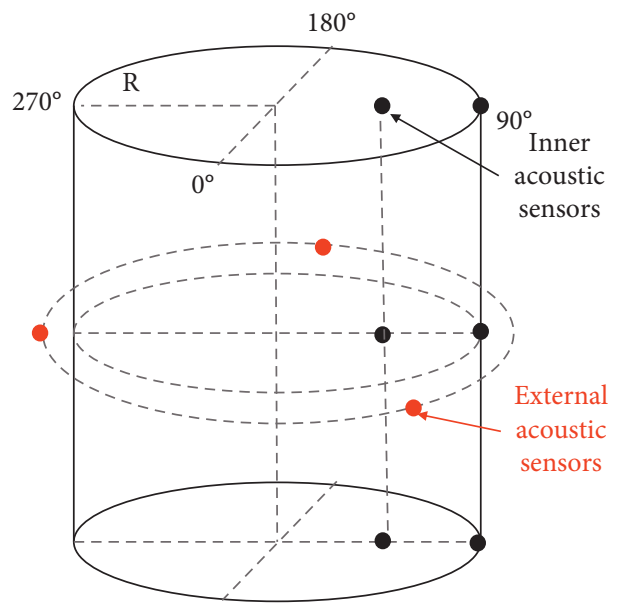

FIGURE 3: Schematic diagram of acoustic sensors.

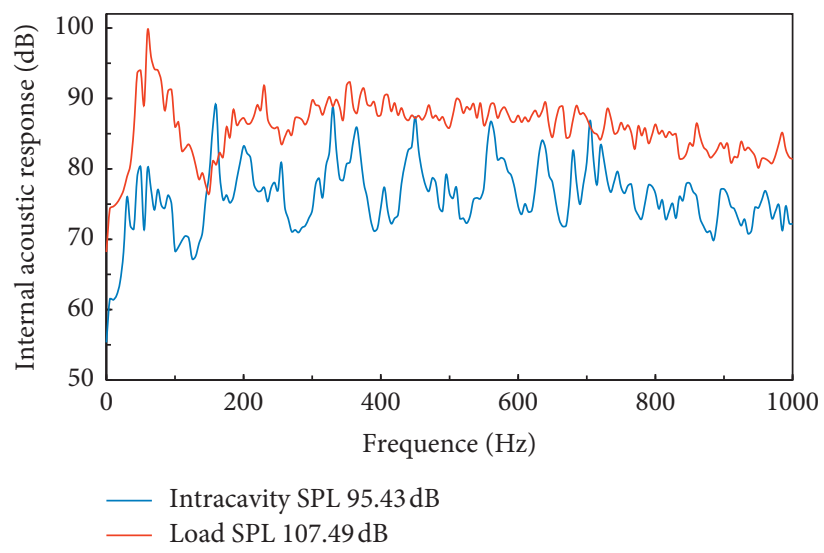

FIGURE 4: SPL inside and outside the cylinder without lining. 
TABLE 1: Material parameters used in the simulation.

\begin{tabular}{lccccccccc}
\hline & $\rho\left(\mathrm{kg} / \mathrm{m}^{3}\right)$ & $\mu$ & $\begin{array}{c}E \\
(\mathrm{GPa})\end{array}$ & $C(\mathrm{~m} / \mathrm{s})$ & $\begin{array}{c}\text { Flow resistivity } \\
\left(\mathrm{Pa} \cdot \mathrm{s} \cdot \mathrm{m}^{-2}\right)\end{array}$ & Porosity & Tortuosity & $\begin{array}{c}\text { Viscous characteristic } \\
\text { length }(\mathrm{mm})\end{array}$ & $\begin{array}{c}\text { Thermal characteristic } \\
\text { length }(\mathrm{mm})\end{array}$ \\
\hline Al & 2710 & 0.34 & 70 & - & - & - & - & - & - \\
Wood & 800 & 0.40 & 2 & - & - & - & - & - & - \\
Air & 1.23 & - & - & 346 & - & - & - & - & - \\
MF & 9.38 & - & - & - & 13000 & 0.97 & 1.05 & 0.064 & 0.207 \\
\hline
\end{tabular}

as 24 rows of plane waves uniformly distributed in the cylinder shell) was adopted in the simulation as shown in Figure 5, and the value is consistent with the experimental measurement through the load function set. The polarization number was set to be 2 . In the inner acoustic field, a field point consistent with the test position of the experimental acoustic sensor is established to output the sound pressure response value of the corresponding field point. And the corresponding SPL spectrum diagram was also obtained, which was then compared with the experimental data to verify the finite element model in this frequency band.

\subsection{Simulation Results and Validation}

3.2.1. Simulation Model Validation. A cylindrical sound cavity model was established according to the model used in the experiment, with the sound cavity height $L$ and radius $a$. Assuming $r=a$ is a rigid boundary condition, the normal frequency of a cylindrical sound cavity is [20]

$$
\frac{\omega_{l m n}^{2}}{c^{2}}=\frac{k_{m n}^{2}}{a^{2}}+\frac{l^{2} \pi^{2}}{L^{2}}
$$

where $c$ is the sound velocity, $l, m$, and $n$ are the orders of natural frequency at the axial, radial, and circumferential modes, $k_{m n}^{2}+k_{z}^{2}=\omega^{2} / c^{2}, k_{m n}$ is the $n$ order solution of $J_{m}^{\prime}\left(K_{m n} r\right)=0$, and $J_{m}(x)$ is the $m$-order Bessel function.

When the sound cavity is lined with MF of thickness $b$, space can be divided into two parts: one is a cylindrical cavity and the other part is the annular cylindrical MF, and the

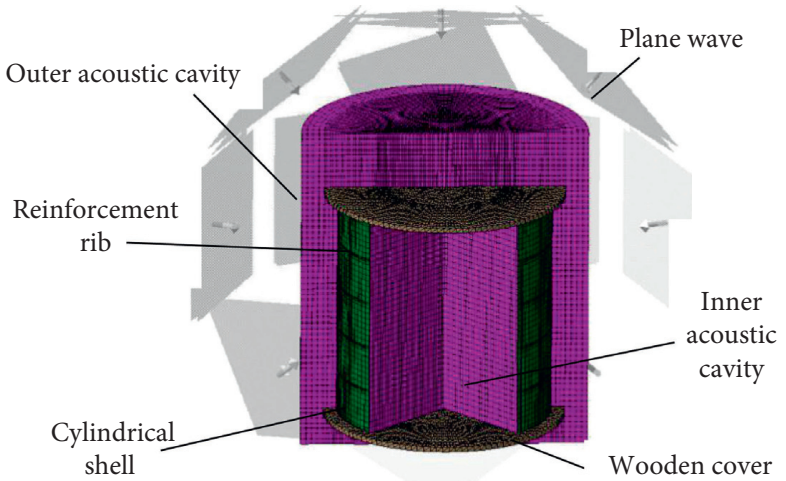

FIgURE 5: Finite element model of cylindrical shell.

solution of the sound pressure equation in cylindrical coordinates is

$$
\begin{aligned}
p_{m}^{\prime}(r, \theta, z)= & A_{m}\left(H_{m}^{(1)}\left(q_{m n} r\right)+R_{m} H_{m}^{(2)}\left(q_{m n} r\right)\right) \\
& \cos (m \theta-\varphi) \cos q_{z} z e^{j \omega t}
\end{aligned}
$$

where $q_{m n}$ is the radial and circumferential wave number $(\mathrm{WN})$ in MF, $q_{z}$ is the axial WN in MF, $H_{m}^{(1)}=J_{m}+j Y_{m}$ and $H_{m}^{(2)}=J_{m}-j Y_{m}$ are the primary and secondary Hankel functions. Similar to planar $\mathrm{WN}, H_{m}^{(1)}$ and $H_{m}^{(2)}$ represent the $m$-order outside and incident waves.

Based on the rigid boundary condition at $r=a$, the normalized acoustic admittance at $r=r_{b}=a-b$ could be obtained as

$$
\eta^{\prime}(\omega)=\rho_{0} c \frac{u_{r m}(a-b)}{p_{m}^{\prime}(a-b)}=j \zeta \frac{q_{m n}}{q} \frac{J_{m}^{\prime}\left[q_{m n}(a-b)\right] Y_{m}^{\prime}\left(q_{m n} a\right)-J_{m}^{\prime}\left(q_{m n} a\right) Y_{m}^{\prime}\left[q_{m n}(a-b)\right]}{J_{m}\left[q_{m n}(a-b)\right] Y_{m}^{\prime}\left(q_{m n} a\right)-J_{m}^{\prime}\left(q_{m n} a\right) Y_{m}\left[q_{m n}(a-b)\right]},
$$

where $\zeta=\rho_{0} c / \sqrt{\rho K}$ is the characteristic impedance ratio between air and $\mathrm{MF}$, and $q$ is the total propagation constant in the porous material.

When $r=r_{b}$, the sound pressure and velocity in air and MF should be continuous, so $v_{r m}(a-b)=v_{m}^{\prime}(a-b)$ and $p_{m}(a-b)=p_{m}^{\prime}(a-b)$, and then, the equation can be obtained as follows:

$$
\eta^{\prime}(\omega)=j \frac{k_{m n}}{k} \frac{J^{\prime}\left[k_{m n}(a-b)\right]}{J_{m}\left[k_{m n}(a-b)\right]},
$$

where $k_{m n}$ is the propagation component of internal wave in air, and $k_{m n}^{2}+k_{z}^{2}=k^{2}=\omega^{2} / c^{2}$. Since air and foam material must have the same wave velocity at $z$-direction, their WN must be identical as well, so $q_{m n}^{2}+q_{z}^{2}=q^{2}=\omega^{2} /(k / \rho)$. The equation could be obtained as

$$
k_{z}^{2}=q_{z}^{2}=k^{2}-k_{m n}^{2}=q^{2}-q_{m n}^{2} .
$$

So the normal frequency of the cylindrical sound cavity lining MF in the cavity could be obtained as

$$
\omega_{\text {lmn }}{ }^{\prime}=k c_{0} \text {. }
$$

The results of the theoretical values were compared with simulation values of the normal frequency of the cylindrical cavity with no lining and lining $40 \mathrm{~mm} \mathrm{MF}$, as given in 
Table 2. The maximum error of simulated results is $0.6 \%$ and $1.4 \%$ for the shell with and without MF, which verifies the correctness of the finite element model.

3.2.2. Comparison of Simulation and Experimental Acoustic Response of the Sound Cavity. Based on the finite element simulation model, the SPL spectrum in the cylindrical shell cavity was simulated and compared with the experimental test. The results are shown in Figure 6.

It can be found that the experimental results are identical to simulation computation results within the frequency range of $100-400 \mathrm{~Hz}$. Several peaks can also be observed, which are caused by the resonance inside the sound cavity near the resonant frequency of the sound cavity. In terms of overall SPL, the simulation and test results of the empty cavity and cavity lining $40 \mathrm{~mm}$ MF only differ $1.9 \%$ and $1.6 \%$. And it can be seen that the response peak of the noise is shifted to the left near the normal frequency after lining $40 \mathrm{~mm}$ MF. This is because the propagation velocity of sound waves in air is higher than lining MF. After lining $40 \mathrm{~mm}$ $\mathrm{MF}$, the continuity of sound waves on the contact surface of air and MF makes the number of waves in both of them the same. The axial normal frequency of the sound cavity decreases as a result. The agreement between the experimental and numerical results proves the correctness of the finite element model.

\section{Results and Discussion}

To further study the law of vibration and noise reduction of acoustic overburden, the finite element simulation method was used to study the influence law of cylindrical shell STL of different thickness MF lining positions and different thickness MF under the same specific ratio in midlow frequency, and the specific ratio refers to the ratio of MF lining mass to total structural mass.

\subsection{Different Thickness MF Lining Position on the Performance} of STL. After validation of the finite element simulation method, the influence of different thickness MF lining positions on the performance of STL was investigated. First, it is necessary to get the quality of cylindrical shell structure and the whole MF lining. The total quality of cylindrical shell structure was measured to be $45.68 \mathrm{~kg}$. If the shell is fully covered, the total mass of MF is $0.62 \mathrm{~kg}, 1.04 \mathrm{~kg}$, and $1.24 \mathrm{~kg}$ for the thickness $20 \mathrm{~mm}, 30 \mathrm{~mm}$, and $40 \mathrm{~mm} \mathrm{MF}$. However, considering the limitation that the mass of the covering layer is no more than $2 \%$ of the total mass of the structure, i.e., $0.91 \mathrm{~kg}$ for this work, the maximum lining rate for the case of $30 \mathrm{~mm}$ and $40 \mathrm{~mm} \mathrm{MF}$ was limited at $88 \%$ and $73 \%$, respectively.

As shown in Figure 7, the cylindrical shell is divided into 5 layers, which are numbered from top to bottom 1-5 in sequence. Within the frequency range of $100-400 \mathrm{~Hz}$, the wavelength of sound waves is $0.865-3.46 \mathrm{~m}$, which is greater than the height of each layer of the sound cavity $0.225 \mathrm{~m}$. For this case, if the lining rate is too high, the location distribution of MF in the cavity has little influence on the STL; if the lining rate is too small, the noise reduction effect in the cavity is poor, which makes the difference of STL caused by the location distribution without lining materials difficult to distinguish. Based on the above, 3 layers of MF were selected from the 5 layers, that is, the lining rate of MF was $60 \%$. Therefore, there are altogether 10 lining schemes of different lining types. Considering the response mode of the sound cavity of cylindrical shell, 4 lining schemes of symmetrical or antisymmetric lining types were selected: 1-2-3, 2-3-4, 3-4-5, and 1-3-5. Based on the finite element software, the corresponding finite element models were established for the four types of lining, respectively. In the acoustic finite element analysis module of LMS Virtual.Lab software, the acoustic cavity response of the cylindrical shell in the frequency range of 100-400 Hz under different thicknesses was calculated.

4.1.1. Influence of Different Lining Position of Lining $20 \mathrm{~mm}$ MF on STL. According to the four lining types selected above, lining $20 \mathrm{~mm} \mathrm{MF}$ is at the corresponding position and establishing the corresponding finite element model. The acoustic grid of the lining MF was defined as the type of porous material, and the acoustic cavity response of the cylindrical shell was simulated within the frequency range of $100-400 \mathrm{~Hz}$. The result is shown in Figure 8.

It can be seen from the figure that the SPL curves of the four lining types are basically the same, which means the influence of the four different lining types on the STL of the structure is negligible. Between the normal frequency of $312 \mathrm{~Hz}$ and $348 \mathrm{~Hz}$, the peak value is slightly different. From the analysis of the total SPL, the four lining types of 1-2-3, 23-4, 3-4-5, and 1-3-5 are, respectively, $88.99 \mathrm{~dB}, 88.86 \mathrm{~dB}$, $88.45 \mathrm{~dB}$, and $89.00 \mathrm{~dB}$. The maximum difference of the overall SPL in the cavity is only $0.55 \mathrm{~dB}$, indicating that the different lining positions of lining $20 \mathrm{~mm}$ MF have little influence on the STL of the cylindrical shell.

\subsubsection{Influence of Different Lining Position of Lining $30 \mathrm{~mm}$} MF on STL. According to the above method, the acoustic cavity response of the cylinder shell lining $30 \mathrm{~mm}$ was simulated within the frequency range of $100-400 \mathrm{~Hz}$. The result is shown in Figure 9.

It can be seen from the figure that the SPL curves of the four lining types are basically the same within the frequency range of $100-260 \mathrm{~Hz}$. However, to some extent, they are different within the frequency range of 260-400 Hz, especially the 3-4-5 lining scheme, there is a significant decrease in the peak value of the SPL corresponding to the normal frequency of $312 \mathrm{~Hz}$. The total SPL of four lining types of 1-2-3, 2-3-4, 3-4-5, and 1-3-5 are, respectively, $87.31 \mathrm{~dB}, 87.08 \mathrm{~dB}, 86.59 \mathrm{~dB}$, and $87.22 \mathrm{~dB}$; among them, the difference of the STL of 1-2-3, 2-3-4, and 1-2-3 lining schemes are only $0.23 \mathrm{~dB} ; 3-4-5$ lining scheme can be further increased by $0.49-0.72 \mathrm{~dB}$, but the effect is not significant, which shows that the different location of $30 \mathrm{~mm}$ MF lining to the influence of the cylindrical shell STL is still small. 
TABLE 2: Comparison between theoretical and simulation values of normal frequency.

\begin{tabular}{lcccc}
\hline \multirow{2}{*}{ Mode } & \multicolumn{2}{c}{ Without MF lining $(\mathrm{Hz})$} & \multicolumn{2}{c}{$40 \mathrm{~mm} \mathrm{MF} \mathrm{lining}(\mathrm{Hz})$} \\
& Theory & Simulation & Theory & 138 \\
$(1,0,0)$ & 153.8 & 153.8 & 137.9 & 180.2 \\
$(0,0,1)$ & 193.5 & 195.0 & 223.3 & 224.0 \\
$(1,0,1)$ & 248.4 & 248.1 & 274.3 & 275.8 \\
$(2,0,0)$ & 307.6 & 307.6 & 285.0 & 287.7 \\
$(0,0,2)$ & 321.0 & 323.1 & 316.4 & 320.9 \\
$(1,0,2)$ & 355.9 & 357.8 & 319.3 & 323.7 \\
$(2,0,1)$ & 363.4 & 365.7 & & \\
\hline
\end{tabular}

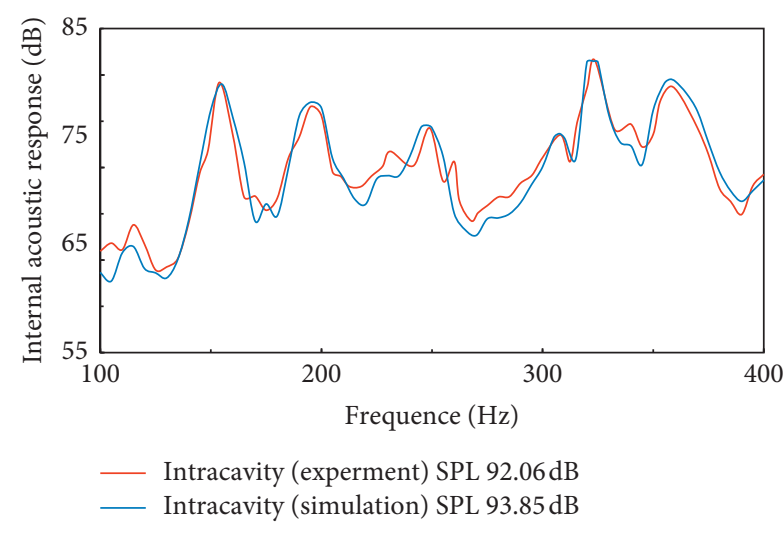

(a)

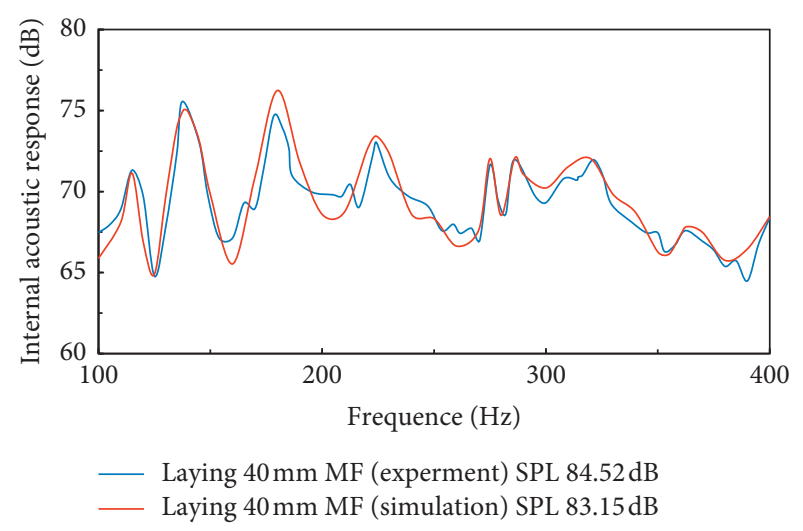

(b)

FIgUre 6: (a) Comparison of simulation analysis and test of the cavity response without MF lining. (b) Comparison of simulation analysis and test of the cavity response with $40 \mathrm{~mm}$ MF lining.

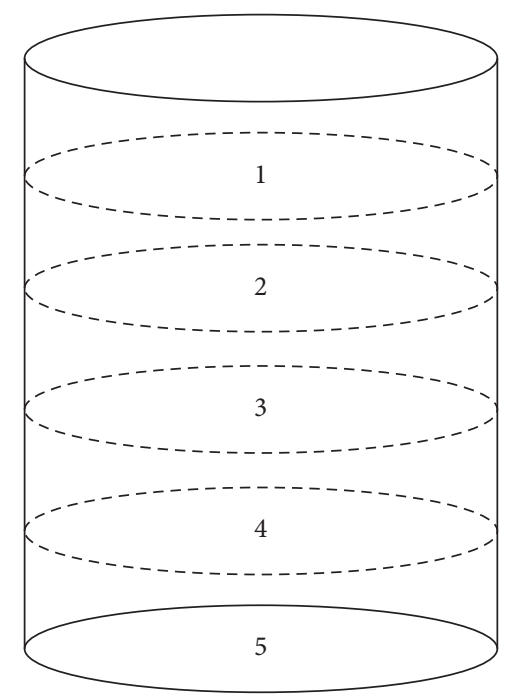

Figure 7: Schematic diagram of layered ring stiffener of cylindrical shell.

4.1.3. Influence of Different Lining Position of Lining $40 \mathrm{~mm}$ $M F$ on STL. In the same way, the frequency spectrum of SPL with different lining schemes of lining $40 \mathrm{~mm} \mathrm{MF}$ is calculated by simulation. The result is shown in Figure 10.

It can be seen from the figure that the SPL curves of the four lining types are basically the same within the frequency range of $100-260 \mathrm{~Hz}$. However, the SPL curves are greatly different in within the frequency range of $260-400 \mathrm{~Hz}$. In particular, the 3-4-5 lining scheme significantly reduces the STL within the frequency range of $275-325 \mathrm{~Hz}$ compared with other schemes. The total SPL of four lining types of 1-2$3,2-3-4,3-4-5$, and 1-3-5 are, respectively, $85.62 \mathrm{~dB}$, $85.23 \mathrm{~dB}, 84.63 \mathrm{~dB}$, and $85.45 \mathrm{~dB}$; among them, the difference of the STL of 1-2-3, 2-3-4, and 1-2-3 lining schemes are 


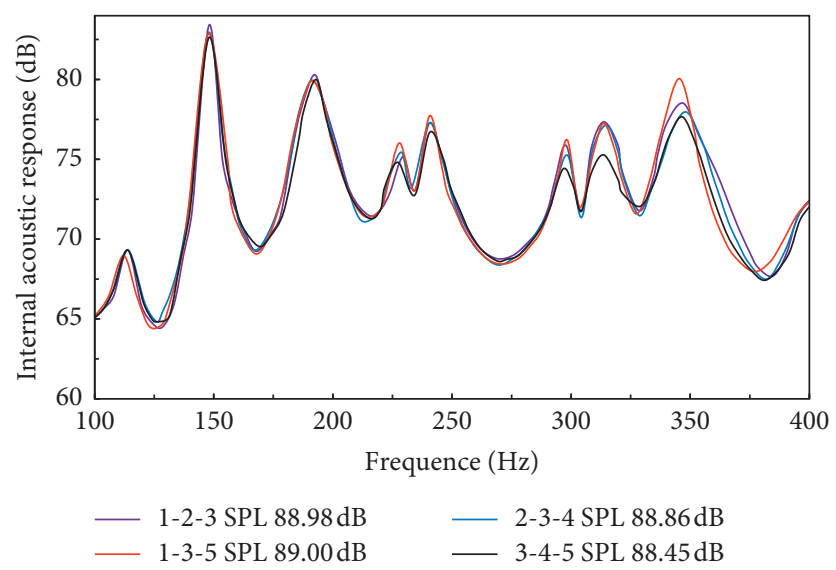

Figure 8: The sound pressure spectrum in the cavity of different lining schemes lining $20 \mathrm{~mm}$ MF.

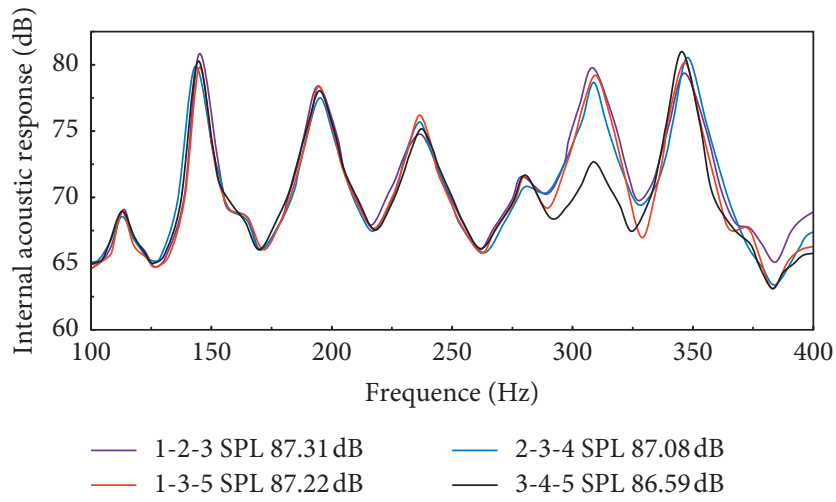

FigurE 9: The sound pressure spectrum in the cavity of different lining schemes lining $30 \mathrm{~mm}$ MF.

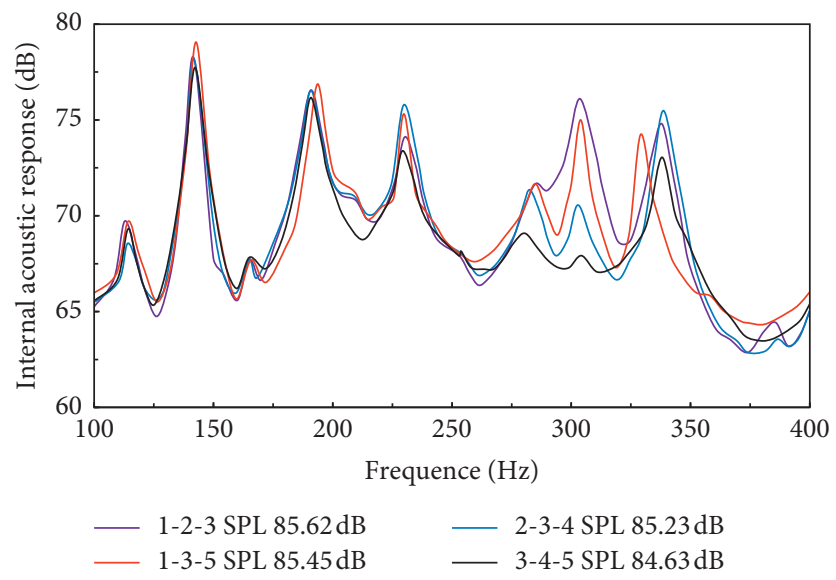

FIgURE 10: The sound pressure spectrum in the cavity of different lining schemes lining $40 \mathrm{~mm}$ MF.

only $0.39 \mathrm{~dB} ; 3-4-5$ lining scheme can be further increased by $0.60-0.99 \mathrm{~dB}$, indicating that the different lining positions of $40 \mathrm{~mm}$ MF lining have evident effect influence on the cylindrical shell STL. Compared with lining $20 \mathrm{~mm}$ and $30 \mathrm{~mm} \mathrm{MF}$, the STL of the cylinder shell with $40 \mathrm{~mm}$ MF was slightly increased in different lining schemes, among which the noise reduction effect of the 3-4-5 lining scheme was better, which could be seen to be caused by its better noise reduction performance at the nondegenerate frequency.

4.2. Influence of Different Thickness $M F$ under the Same Specific Gravity Ratio. When the specific proportion of lining material in the cavity is certain, there are two lining schemes to optimize the STL of the shell: one is to reduce the 


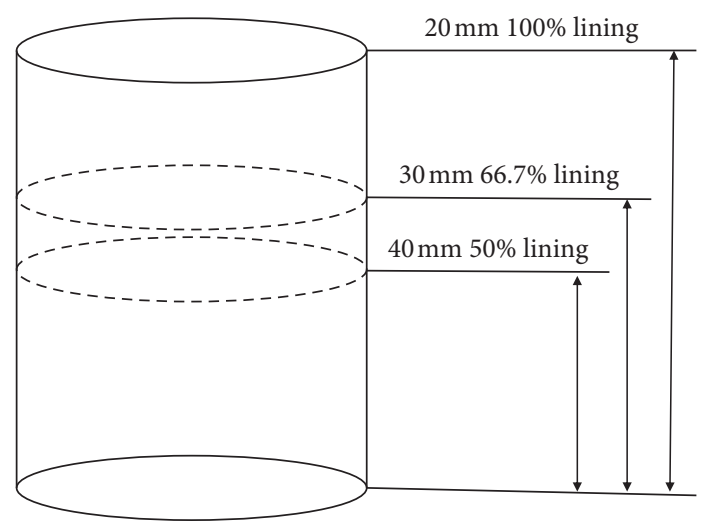

Figure 11: Different lining schemes under the same specific gravity ratio.

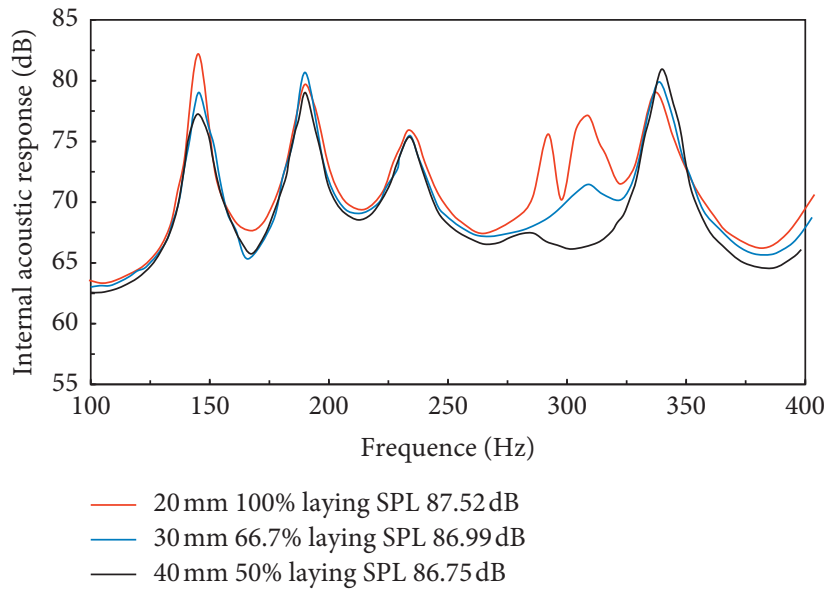

FIgURE 12: The sound pressure spectrum in the cavity of different lining schemes under the same specific gravity ratio.

thickness of lining material to improve the area of lining and the second is to adjust the lining location of sound absorption and lay thicker sound absorption materials at this location. To select a more reasonable lining method, the lining rate of $20 \mathrm{~mm}, 30 \mathrm{~mm}$, and $40 \mathrm{~mm} \mathrm{MF}$ is set to be $100 \%, 66.70 \%$, and $50 \%$, respectively, which ensures the specific ratio of the three lining materials is the same and do not exceed the stipulated limit.

The research in Section 4.1 indicates the optimal solution of MF lining of the cylindrical shell is 3-4-5 type, i.e., lining MF from bottom to top, as shown in Figure 11. Same as the method in Section 4.1, establish finite element models of different schemes and simulate acoustic cavity response of the cylindrical shell within the frequency range of $100-400 \mathrm{~Hz}$. The result is shown in Figure 12.

It can be seen from the figure that within the frequency range of $280-320 \mathrm{~Hz}$, the wavelength of sound waves is similar to the length of the sound cavity, and lining $40 \mathrm{~mm}$ (50\% laying) $\mathrm{MF}$ has the best noise reduction effect. However, in the frequency range of $100-280 \mathrm{~Hz}$ and $320-400 \mathrm{~Hz}$, the trends of the SPL curve of different lining schemes are the same; only the response peaks corresponding to the normal frequency are different. From the perspective of overall SPL, the total SPL of three lining schemes (20 mm, 100\% laying; $30 \mathrm{~mm}, 66.7 \%$ laying; and
$40 \mathrm{~mm}, 50 \%$ laying) is $87.52 \mathrm{~dB}, 86.99 \mathrm{~dB}$, and $86.75 \mathrm{~dB}$. Compared to the results in Figure 6(a), STL of the three lining scheme is $6.33 \mathrm{~dB}, 6.86 \mathrm{~dB}$, and $7.10 \mathrm{~dB}$. The result shows that, under the same specific gravity ratio, the thicker the layer is, the better the noise reduction effect at midlow frequency is. It also can be seen that the increment of STL decreases with the increase of the thickness of the layer. Therefore, within the limited specific gravity ratio of lining material in the cavity, the thicker material is suggested to be laid in the position with the highest noise reduction efficiency to achieve the optimal noise reduction effect of midlow frequency.

\section{Conclusion}

This study takes the aluminum cylindrical shell lining MF as the research object, establishes the finite element simulation model of aluminum and aluminum cylindrical shell lining MF, and verifies the correctness of the finite element model within the frequency range of $100-400 \mathrm{~Hz}$. Aiming at the noise reduction problem of the cylindrical shell, the influence of lining position of different thickness MF is first studied. Based on the results, the optimal laying scheme under the same specific gravity ratio is then investigated, and the following conclusions are obtained: 
(1) When the laying rate of MF lining is $60 \%$ and the thickness of MF is greater than $6 \%$ of the cavity radius, the performance of STL is obviously improved with thicker MF despite different laying position. Among them, the STL of the 3-4-5 lining scheme can be further increased by $0.49-0.99 \mathrm{~dB}$ compared with other lining schemes.

(2) At the same specific gravity ratio, the noise reduction amount in the midlow frequency in the cavity increases with the increase of material thickness, but the increment decreases. Moreover, the difference of the noise reduction effect is mainly reflected in the frequency band where sound wavelength and sound cavity length are close to each other.

(3) At midlow frequency, when the internal lining materials are limited by a gravity ratio, it is suggested to lay thicker materials in the position with high noise reduction efficiency to achieve a better noise reduction effect, rather than choosing thinner materials for more area of lining

\section{Data Availability}

The data generated or analyzed during this study are included within the article.

\section{Conflicts of Interest}

The authors declare that there are no conflicts of interest.

\section{Acknowledgments}

The project was supported by the open foundation of Hubei Key Laboratory of Theory and Application of Advanced Materials Mechanics, Wuhan University of Technology (TAM202003), and General scientific research projects of Wuhan Polytechnic University (2020Y11).

\section{References}

[1] D. Li and T. Cheng, "Development prospect of China's newgeneration launch vehicle," China Engineering Science, vol. 8, no. 1, pp. 33-38, 2006.

[2] Q. Shi, S. Ando, K. Murakami et al., "Acoustic analysis of H-IIA launch vehicle fairing using SEA," in Proceedings of the Fourth International Symposium Environmental Testing for Space Programmes, vol. 467, pp. 425-430, Liege, Belgium, June 2001.

[3] S. A. Lane, S. Kennedy, and R. Richard, "Noise transmission studies of an advanced grid-stiffened composite fairing," Journal of Spacecraft and Rockets, vol. 44, no. 5, pp. 1131-1139, 2012.

[4] M. H. Asadijafari, M. R. Zarastvand, and R. Talebitooti, "The effect of considering pasternak elastic foundation on acoustic insulation of the finite doubly curved composite structures," Composite Structures, vol. 256, 2020.

[5] S. Valvano, A. Alaimo, and C. Orlando, "Sound transmission analysis of viscoelastic composite multilayered shells structures," Aerospace, vol. 6, no. 6, p. 69, 2019.
[6] S. Chonan and H. Koriyama, "Sound insulation characteristics of finite length orthotropic shells," Journal of Sound and Vibration, vol. 126, no. 3, pp. 525-532, 1988.

[7] S. Ghinet, N. Atalla, and H. Osman, "Diffuse field transmission into infinite sandwich composite and laminate composite cylinders," Journal of Sound and Vibration, vol. 289, no. 4, pp. 745-778, 2006.

[8] X. L. Yao, X. Z. Wang, and L. Q. Sun, "Acoustic transmission analysis of cylindrical shell with viscoelastic layer coating," Journal of Vibration and Shock, vol. 31, no. 15, pp. 116-120, 2012.

[9] H. Denli and J. Q. Sun, "Structural-acoustic optimization of sandwich structures with cellular cores for minimum sound radiation," Journal of Sound and Vibration, vol. 301, no. 1, pp. 93-105, 2007.

[10] H. Denli and J. Q. Sun, "Structural-acoustic optimization of sandwich cylindrical shells for minimum interior sound transmission," Journal of Sound and Vibration, vol. 316, no. 1, pp. 32-49, 2008.

[11] R. Talebitooti, M. R. Zarastvand, and H. D. Gohari, "The influence of boundaries on sound insulation of the multilayered aerospace poroelastic composite structure," Aerospace Science and Technology, vol. 80, pp. 452-471, 2018.

[12] S. Valvano, A. Alaimo, and C. Orlando, "Analytical analysis of sound transmission in passive damped multilayered shells," Composite Structures, vol. 253, 2020.

[13] T. Engberg and U. A. Korde, "Modeling of the acoustic response of payload bays within launch vehicle fairings," Journal of Spacecraft and Rockets, vol. 50, no. 2, pp. 423-432, 2012.

[14] M. Chen, D. Luo, P. Xu et al., "Analysis of characteristics of sound radiation from double cylindrical shell coated with viscoelastic layer," Chinese Journal of Acoustics, vol. 28, no. 1, pp. 45-57, 2004.

[15] B. Li, J. Li, S. Yan, W. Yan, and X. He, "Experiment and simulation analysis on noise attenuation of Al/MF cylindrical shells," Shock and Vibration, vol. 2017, Article ID 6980501, 8 pages, 2017.

[16] B. Li, J. Li, S. Yan, L. Ju, and X. He, "Impacts of porous material fluid bulk properties on noise attenuation performance of cylinder shell structure based on finite element model," Concurrency and Computation: Practice and Experience, vol. 31, no. 10, pp. e4714.1-e4714.9, 2019.

[17] B. Li, X. Chen, S. Yan, W. Yan, and X. He, "Theoretical and experimental study on effect of melamine foam lining on acoustic characteristics of a cylindrical cavity," Results in Physics, vol. 13, 2019.

[18] W. Yan, B. Li, S. Yan et al., "Experiment and simulation analysis on noise reduction of cylindrical shells with viscoelastic material," Results in Physics, vol. 14, 2019.

[19] H. D. Gohari, M. R. Zarastvand, and R. Talebitooti, "Acoustic performance prediction of a multilayered finite cylinder equipped with porous foam media," Journal of Vibration and Control, vol. 26, no. 11-12, 2020.

[20] U. Ingard, Noise Reduction Analysis (Physics), Infinity Science Press, Hingham, MA, USA, 2010. 